\title{
Prognostic and diagnostic significance of copeptin in acute exacerbation of chronic obstructive pulmonary disease and acute heart failure: data from the ACE 2 study
}

Jacob A. Winther ${ }^{1,2^{*}}$, Jon Brynildsen ${ }^{1,2}$, Arne Didrik Høiseth ${ }^{1,2}$, Heidi Strand ${ }^{4}$, Ivar Følling ${ }^{1,2}$, Geir Christensen ${ }^{3}$, Ståle Nygård ${ }^{5}$, Helge Røsjø $\varnothing^{1,2}$ and Torbjørn Omland $d^{1,2}$

\begin{abstract}
Background: Copeptin is a novel biomarker that predicts mortality in lower respiratory tract infections and heart failure (HF), but the diagnostic value of copeptin in acute dyspnea and the prognostic significance of copeptin in acute exacerbation of chronic obstructive pulmonary disease (AECOPD) is not clear.

Method: We determined copeptin and NT-proBNP concentrations at hospital admission in 314 patients with acute dyspnea who were categorized by diagnosis. Survival was registered after a median follow-up of 816 days, and the prognostic and diagnostic properties of copeptin and NT-proBNP were analyzed in acute HF $(n=143)$ and AECOPD ( $n=84)$ separately.

Results: The median concentration of copeptin at admission was lower in AECOPD compared to acute HF (8.8 [5. $2-19.7]$ vs. 22.2 [10.2-47.9]) pmol/L, $p<0.001)$, but NT-proBNP discriminated acute HF from non-HF related dyspnea more accurately than copeptin (ROC-AUC 0.85 [0.81-0.89] vs. 0.71 [0.66-0.77], $p<0.0001$ ). Adjusted for basic risk factors, increased copeptin concentrations predicted mortality in AECOPD (HR per log (In) unit 1.72 [95\% Cl 1.21-2. $45], p=0.003)$ and acute HF (1.61 [1.25-2.09], $p<0.001)$, whereas NT-proBNP concentrations predicted mortality only in acute HF (1.62 [1.27-2.06], $p<0.001)$. On top of a basic model copeptin reclassified a significant proportion of patients into a more accurate risk strata in AECOPD (NRI 0.60 [0.19-1.02], $p=0.004)$ and acute HF (0.39 [0.06-0. 71], $p=0.020$ ).

Conclusion: Copeptin is a strong prognostic marker in both AECOPD and acute HF, while NT-proBNP concentrations predict mortality only in patients with acute HF. NT-proBNP levels are superior to copeptin levels to diagnose acute HF in patients with acute dyspnea.
\end{abstract}

Keywords: Copeptin, NT-proBNP, COPD, Heart failure, Epidemiology, Dyspnea, Hyponatremia, Vasopressin, Antidiuretic hormone

\footnotetext{
* Correspondence: j.a.winther@medisin.uio.no

${ }^{1}$ Division of Medicine, Akershus University Hospital, Lørenskog, Norway

${ }^{2}$ Institute of Clinical Medicine, University of Oslo, Oslo, Norway

Full list of author information is available at the end of the article
} 


\section{Background}

Acute dyspnea is a major symptom of cardiac and pulmonary pathology frequently leading to hospital admission. Systemic biomarkers, such as cardiac troponins and B-type natriuretic peptides (BNPs), are useful tools in the diagnostic work-up and risk stratification of patients with acute coronary heart disease and heart failure (HF) [1-3], but the clinical application of biochemical markers in acute exacerbation of chronic obstructive pulmonary disease (AECOPD) is more uncertain due to lack of validation of potential candidates [4].

Copeptin is a novel biomarker that could prove helpful in the differential diagnosis and risk evaluation of patients with acute dyspnea. The function of copeptin is unknown, but the molecule is derived from the 39-amino acid C-terminal fragment of the arginine-vasopressin (AVP) precursor molecule, prepro-vasopressin. Copeptin and AVP are released in equimolar amounts and plasma levels correlate well [5]. Thus, copeptin plasma concentrations are most likely regulated by the same mechanisms that have been established for AVP. Under normal conditions AVP secretion is regulated according to plasma osmolality by osmoreceptors in the hypothalamus, but several strong non-osmotic pathways also exist [6]. In particular, arterial under-filling, as observed during heart failure, stimulates AVP secretion via baroreceptors in the carotid sinus and the aortic arch [7]. Pulmonary disorders, including COPD are also associated with elevated AVP levels, possibly due to impaired gas exchange or activation of baroreceptors $[8,9]$, but the mechanism is not clear. In addition, AVP secretion is increased as part of a general stress response [10]. While analytical challenges and stability issues have made reliable measurements of circulating AVP difficult to achieve [11], copeptin is easily measured and stable in plasma and serum for at least 7 days in room temperature and over several freeze and thaw cycles $[5,12]$. The prognostic value of copeptin has already been studied in several medical conditions. Previous studies have found increased copeptin concentrations to be associated with poor prognosis in sepsis and hemorrhagic shock [13, 14], myocardial infarction [15], and chronic HF [16-18]. In patients with lower respiratory tract infections, copeptin predicted mortality more accurately than C-reactive protein (CRP) and leucocyte count [19]. Among patients admitted to hospital with acute dyspnea of various etiologies, copeptin was found to be a strong prognostic marker with superior accuracy compared to BNP and NT-proBNP [20]. The aim of the present study was to compare the prognostic and diagnostic properties of copeptin and N-terminal pro-hormone of BNP (NT-proBNP) in acute HF and AECOPD.

\section{Methods}

\section{Akershus cardiac examination (ACE) 2 study}

The Akershus Cardiac Examination (ACE) 2 Study was designed to assess the diagnostic and prognostic value of circulating biomarkers in patients admitted with acute dyspnea to Akershus University Hospital, Lørenskog, Norway. The primary aim of the ACE 2 study was to analyze the prognostic properties of secretoneurin, and a minimum sample size of 350 patients was originally calculated by power analysis for this purpose [21]. The method of patient recruitment and data collection has also been described in detail previously [22-24]. Patients over the age of 18 years were eligible for inclusion during the first $24 \mathrm{~h}$ of admission if acute dyspnea was the primary cause for hospitalization as evaluated by the emergency department physician. Exclusion criteria were dementia and other causes precluding informed patient consent, disseminated malignant disease, acute myocardial infarction or coronary intervention, major surgery within the last 2 weeks, incomplete study blood sampling, and hemoglobin $<10 \mathrm{~g} / \mathrm{dL}$. Consecutive patients were enrolled between 8 am and $2 \mathrm{pm}$ Monday to Thursday. Clinical information was obtained from physicians on call, hospital records, and directly from the patients by dedicated study personnel who used standardized questionnaires. Echocardiography and spirometry results were registered from hospital records. The ACE 2 study was approved by the Norwegian Regional Committees for Medical and Health Research Ethics (REC) South East (\#5.2008.2832) and conducted in agreement with the Declaration of Helsinki. All participants provided written informed consent prior to study enrolment.

\section{Adjudication of diagnosis and outcome}

The final diagnosis of the index hospitalization was established by two senior physicians working independently of each other, and discordant diagnoses were resolved by consensus. The two members of the adjudication committee had no knowledge of study biomarker levels, but they had access to all medical records, including follow-up data and cardiac biomarker measurements such as NTproBNP and troponin $\mathrm{T}$ that were ordered by the treating physician. Patients were first classified into acute HF and non-HF related dyspnea, and then patients in the non-HF group were evaluated with respect to the AECOPD diagnosis. The acute HF diagnosis was determined by the European Society of Cardiology criteria [25], and the AECOPD diagnosis was based on the criteria defined by the Global initiative for Chronic Obstructive Lung Disease (GOLD) [26]. Discordant diagnoses were resolved by consensus. Survival status was recorded from electronic hospital records, which are synchronized with Statistics Norway, until the end of follow-up November 1st, 2012. 


\section{Laboratory analysis}

Standard biochemical work-up and arterial blood gas measurements were collected at admission. Glomerular filtration rate (GFR) was estimated by the Chronic Kidney Disease Epidemiology Collaboration (CKD-EPI) formula. Study blood sampling was performed by venipuncture and uniformly processed throughout the study period. Copeptin, N-terminal pro-B-type natriuretic peptide (NT-proBNP) and high-sensitivity cardiac troponin $\mathrm{T}$ (hs-TnT) were measured in samples obtained $<24 \mathrm{~h}$ after hospital admission by commercially available assays: B-R-A-H-M-S Kryptor Copeptin assay by Thermo Fisher Scientific Inc., Clinical Diagnostics, BRAHMS GmbH, 16,761 Hennigsdorf, Germany; and proBNP II and troponin $\mathrm{T}$ hs STAT assays by Roche Diagnostics, Penzberg, Germany. Copeptin and hs-TnT were measured in serum while NT-proBNP was analyzed in plasma samples. The copeptin assay had a detection limit of $0.9 \mathrm{pmol} / \mathrm{L}$, a functional sensitivity (inter-analysis variation $<20 \%$ ) above $2.0 \mathrm{pmol} / \mathrm{L}$, and a normal reference range (2.5-97.5 percentile) of 0.9$14.9 \mathrm{pmol} / \mathrm{L}$ for healthy adults.

\section{Statistics}

We report continuous variables as mean ( \pm standard deviation [SD]) or median (quartile [Q] 1-3) depending on variable distribution. Differences between groups were compared by Student's $t$ test or Mann-Whitney $U$ tests as appropriate. Binary data were compared by the chisquare test and are presented as absolute numbers and percentages. Positively skewed variables, including biomarkers, were log transformed by the natural logarithm to approach normal distribution and to reduce the effect of outliers in regression analysis. Variables associated with copeptin concentration were explored by Spearman's rank correlation coefficient (rho) and linear regression analysis, and independent associations were determined by multivariate linear regression using stepwise forward selection of variables. Patient survival stratified by admission copeptin and NT-proBNP quartiles was analyzed by Kaplan-Meier plots and compared by the log-rank test. We identified factors associated with mortality by univariate Cox proportional hazard regression analysis. A basic multivariate Cox model of independent risk factors excluding biomarkers was constructed by stepwise forward selection based on the likelihood ratio criterion. The independent prognostic effect of each biomarker was determined by adjusting for the variables in the basic clinical risk model. The area under receiver operating curves (ROC-AUC) was used to ascertain the diagnostic and prognostic accuracies of biomarkers, while the value of adding biomarkers to the basic clinical risk models was investigated by calculating the category free net reclassification index (NRI). ROC-
AUCs are presented with 95\% confidence interval (CI) computed by bootstrap using 5000 iterations. We considered $p<0.05$ to be statistically significant and statistical analyses were performed using SPSS for Windows version 22.0 (SPSS, Armonk, NY), STATA version 14 (Stata Corp LP, TX, USA), and R 3.3.3 (R Foundation for Statistical Computing, Vienna, Austria). NRI was calculated using the $\mathrm{R}$ package PredictABEL.

\section{Results}

In total, 314 of 468 eligible patients were included in the ACE 2 Study from June 2009 until November 2010. Acute HF was determined to be the primary cause of dyspnea in 143 patients, while 84 patients were diagnosed with AECOPD (Fig. 1). Among patients with dyspnea not related to acute HF or AECOPD $(n=87)$ the most frequent diagnoses were pneumonia (27/87), asthma (16/87), and pulmonary embolism (10/87). Median time from hospitalization to adjudication of diagnosis was 464 days (Q 1-3304-705). The two members of the adjudication committee reached the same diagnosis in 95\% (298/314) of the cases, while the remaining $5 \%(16 / 324)$ were resolved by consensus. The baseline characteristics of acute HF and AECOPD patients were consistent with the respective diagnosis (Table 1). Among patients diagnosed with acute HF, chronic HF was previously recognized in $61 \%$, and $43 \%$ also had a history of COPD. In the AECOPD group, all patients were previously diagnosed with COPD and the prevalence of chronic HF was $11 \%$.

\section{Copeptin concentrations and relation to prognosis}

The prognostic properties of copeptin were analyzed in AECOPD and acute HF separately. After a median follow-up of 2.2 years (813 [356-996] days), 46\% (66/ $143)$ of HF patients and $42 \%$ (35/84) of AECOPD patients had died. According to Kaplan-Meier estimates (Fig. 2) the risk of mortality during follow-up increased among acute HF patients if copeptin or NT-proBNP levels were elevated on hospital admission $(p<0.0001$ by the log-rank test for both biomarkers). In contrast, only copeptin levels were associated with mortality among patients diagnosed with AEOCPD (Fig. 2; $\mathrm{p}<0.0001$ by the log-rank test). After adjustment for basic clinical risk factors, as identified by univariate screening (Additional file 1: Table S2), the risk of dying increased by $72 \%$ in AECOPD (HR 1.72 [1.21-2.45], $p=0.003)$ and $61 \%$ in acute HF (1.61 [1.25-2.09], $p<0.001)$ per $\log (\ln )$ unit increment of copeptin by multivariate Cox analysis (Table 2). In comparison, one $\log (\ln )$ unit increase of NT-proBNP increased the risk of mortality by $62 \%$ in acute HF (1.62 [1.27-2.06], $p<0.001$ ), while no significant predictive effect was found in AECOPD (1.12 [0.88-1.42], $p=0.373)$. Neither copeptin nor NT-proBNP were independently associated 


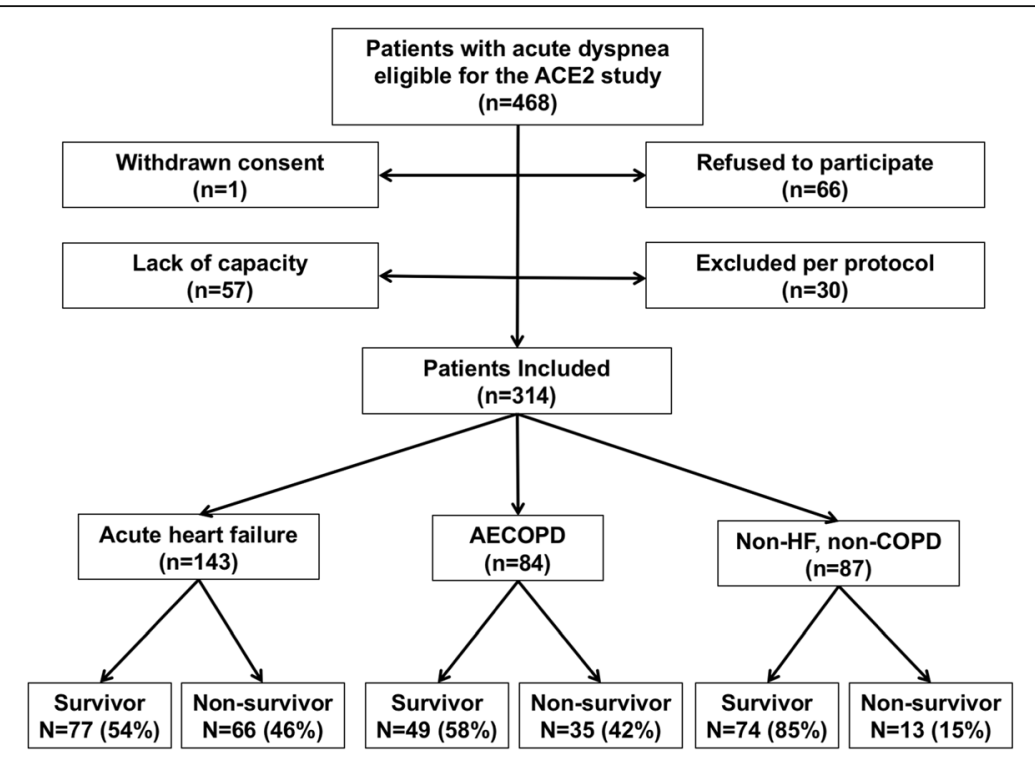

Fig. 1 ACE 2 study flow chart

with mortality in patients with dyspnea that was not related to CODP or HF (Additional file 1: Table S3). When copeptin and NT-proBNP was included in the same model, the predictive effect of copeptin was significant in patients with AECOPD (HR 1.79 [1.20-2.66], $p=0.004)$, but not in patients with acute HF $(1.30$ [0.96-1.76], $p=0.091)$. When copeptin was added to the basic clinical risk model, the category free net reclassification index (NRI) was positive in AECOPD (NRI 0.60 [0.19-1.02], $p=0.004)$ and acute HF (0.39 [0.06-0.71], $p=0.020)$. In the AECOPD group, the predicted risk of mortality decreased in $67 \%$ of survivors and increased in $63 \%$ of non-survivors with the model that included copeptin (Fig. 3). By ROC-AUC analysis, we could not find any statistical difference between the prognostic accuracy of copeptin and NT-proBNP in AECOPD (ROCAUC 0.67 [0.55-0.79] vs. 0.56 [0.44-0.69], $p=0.111$ ) or acute HF (0.66 [0.57-0.75] vs. 0.67 [0.58-0.76], $p=0.695)$. Adding NT-proBNP to the Cox regression model that already included copeptin or vice versa did not significantly alter the overall predictive accuracy of the model, as determined by ROC-AUC or category free NRI, in acute HF.

\section{Copeptin concentrations and relation to diagnosis}

At admission, a large portion of AECOPD patients (35\% [29/84]) and acute HF patients (64\% [91/143] had copeptin concentrations that exceeded the upper reference limit $(14.9 \mathrm{pmol} / \mathrm{L})$ reported for healthy subjects by the manufacturer of the current assay. The median copeptin concentration was significantly higher among acute HF patients compared to patients with AECOPD and other causes of dyspnea (22.2 [10.2-47.9] vs. 8.8 [5.2-19.7] and 8.3 [4.3-18.2] pmol/L), but NT-proBNP discriminated acute HF from non-HF related dyspnea more accurately than copeptin (AUC 0.85 [95\% CI 0.810.89 ] vs. 0.71 [0.66-0.77], $p<0.0001)$. We did not find any significant difference in the concentration of copeptin or NT-proBNP between patients with AECOPD and patients with other causes of dyspnea not related to HF. By multivariate linear regression analysis across all groups (Additional file 1: Table S1) increased copeptin concentrations were independently associated with increased hs-TnT, NT-proBNP, $\mathrm{Na}^{+}$, male gender and reduced eGFR $\left(\mathrm{r}^{2}=0.51\right)$. The correlation between copeptin and individual independent covariates were moderate for NT-proBNP (rho 0.60), hs-TNT (0.55), and eGFR (-0.52); and weak for male gender (0.27) and $\mathrm{Na}^{+}$(0.24). While low $\mathrm{Na}^{+}$levels were associated with lower levels of copeptin (Additional file 1: Figure S1), copeptin concentrations were measurable also among patients with hyponatremia $\left(\mathrm{Na}^{+}\right.$concentrations $<137 \mathrm{mmol} / \mathrm{L})$ in AECOPD (7.6 [2.7-16.0]) and acute HF (18.2 [6.3-52.6] pmol/L).

\section{Discussion}

In this prospective observational study, we found copeptin to be a strong prognostic marker in both AECOPD and acute HF, while NT-proBNP predicted mortality only among acute HF patients. On the other hand, NTproBNP concentrations on admission were superior to copeptin concentrations to separate patients with acute HF from patients with non-HF related dyspnea. 
Table 1 Baseline characteristics

\begin{tabular}{|c|c|c|c|c|}
\hline & $\operatorname{AECOPD}(n=84)$ & Acute HF $(n=143)$ & $\begin{array}{l}\text { Non-HF, non-COPD } \\
(n=87)\end{array}$ & $P^{*}$ \\
\hline Age (years) & $69 \pm 9$ & $75 \pm 11$ & $73 \pm 18$ & $<0.001$ \\
\hline Male sex & $35(42 \%)$ & $90(63 \%)$ & $39(45 \%)$ & 0.002 \\
\hline $\mathrm{BMI}\left(\mathrm{kg} / \mathrm{m}^{2}\right)$ & $24 \pm 6$ & $27 \pm 6$ & $29 \pm 9$ & 0.005 \\
\hline Heart rate (beats/min) & $97 \pm 18$ & $92 \pm 26$ & $91 \pm 22$ & 0.107 \\
\hline MAP $(\mathrm{mmHg})$ & $102 \pm 18$ & $104 \pm 21$ & $99 \pm 16$ & 0.546 \\
\hline Peripheral edema & 31 (37\%) & 77 (54\%) & $16(18 \%)$ & 0.014 \\
\hline NYHA class IV vs. II-III & $47(56 \%)$ & $65(46 \%)$ & $24(28 \%)$ & 0.127 \\
\hline LVEF (\%) & $60(50-60)^{a}$ & $40(30-55)$ & $60(54-60)^{a}$ & $<0.001$ \\
\hline $\mathrm{FEV}_{1} \%$ of predicted & $39 \pm 17$ & n.a ${ }^{b}$ & n.a $a^{b}$ & \\
\hline $\mathrm{FEV}_{1} / \mathrm{FVC}(\%)$ & $47 \pm 15^{a}$ & n.a ${ }^{b}$ & n.. ${ }^{b}$ & \\
\hline Current smoker & $28(33 \%)$ & $30(21 \%)$ & $27(31 \%)$ & 0.039 \\
\hline Diabetes & $9(11 \%)$ & $43(30 \%)$ & $16(18 \%)$ & 0.001 \\
\hline Chronic heart failure & $9(11 \%)$ & $87(61 \%)$ & $5(6 \%)$ & $<0.001$ \\
\hline Coronary artery disease & $23(27 \%)$ & $78(55 \%)$ & $10(12 \%)$ & $<0.001$ \\
\hline Hypertension & $26(31 \%)$ & $69(48 \%)$ & $25(29 \%)$ & 0.011 \\
\hline COPD & $84(100 \%)$ & $61(43 \%)$ & $10(12 \%)$ & $<0.001$ \\
\hline Beta-blocker & 31 (37\%) & 89 (62\%) & 19 (22\%) & $<0.001$ \\
\hline ACEi/ARB & $27(32 \%)$ & $87(61 \%)$ & $25(29 \%)$ & $<0.001$ \\
\hline Diuretic therapy & 33 (39\%) & $104(73 \%)$ & $23(27 \%)$ & $<0.001$ \\
\hline $\mathrm{K}^{+}(\mathrm{mmol} / \mathrm{L})$ & $4.3 \pm 0.5$ & $4.4 \pm 0.6$ & $4.2 \pm 0.5$ & 0.667 \\
\hline $\mathrm{Na}^{+}(\mathrm{mmol} / \mathrm{L})$ & $138(134-140)$ & $139(136-141)$ & $138(136-140)$ & 0.050 \\
\hline eGFR (mL/min) & $82 \pm 20$ & $61 \pm 24$ & $87 \pm 31$ & $<0.001$ \\
\hline $\mathrm{CRP}(\mathrm{mg} / \mathrm{L})$ & $26(6-50)$ & $13(5-35)$ & $16.5(1-95)$ & 0.019 \\
\hline hs-TnT (ng/L) & $18(9-28)$ & $38(22-75)$ & $9(3-23)$ & $<0.001$ \\
\hline NT-proBNP (pg/mL) & $379(171-1010)$ & 3600 (1601-8396) & $280(88-1293)$ & $<0.001$ \\
\hline Copeptin (pmol/L) & $8.8(5.2-19.7)$ & $22.2(10.2-47.9)$ & $8.3(4.3-18.2)$ & $<0.001$ \\
\hline
\end{tabular}

Continuous variables are presented as mean \pm standard deviation or median (quartile 1-3). Binary variables are presented as absolute numbers and percentages Abbreviations: ACEi angiotensin-converting-enzyme inhibitor, AECOPD Acute exacerbation of chronic obstructive pulmonary disease, ARB angiotensin II receptor blocker, BMI Body mass index, CRP C-reactive protein, eGFR estimated glomerular filtration rate (CKD-EPI), FEV1 forced expiratory volume in one second, FVC forced vital capacity, HF heart failure, $h s-T n T$ high sensitivity troponin T, LVEF left ventricular ejection fraction, n.a. not applicable, NT-proBNP N-terminal pro-B-type natriuretic peptide, NYHA New York Heart Association, MAP Mean arterial pressure

" $P$ for difference between AECOPD and acute HF

${ }^{\text {a Missing data }}>10 \%$

${ }^{\mathrm{b}}$ Missing data $>85 \%$

Sparse data exist concerning the prognostic value of copeptin in AECOPD. One previous study found that copeptin predicted mortality in a mixed population with lower respiratory tract infection, but the majority of patients in that study suffered from community-acquired pneumonia, and only 60 of 543 patients had AECOPD [19]. Another study found increasing levels of copeptin to be associated with poor prognosis in AECOPD when using a composite outcome of re-hospitalization and death [27]; however, composite outcomes are associated with uncertainty with respect to the association with individual components of the outcome [28]. A recent multicenter study found that copeptin measured in stable-state COPD predicted two-year mortality independently of selected pulmonary risk factors, and recommended a new risk assessment index including copeptin [29]. Finally, a newly published multicenter study of AECOPD patients did not find any association between copeptin and a short-term (30 days) composite outcome that included mortality, transfer to the intensive care unit, or a new visit to the emergency room. Notably, patients who required immediate intensive care unit monitoring and/or assisted ventilation (invasive or non-invasive) were excluded from the study and only 14 of 277 included patients died [30]. To clarify the prognostic properties of copeptin with respect to mortality in 
a
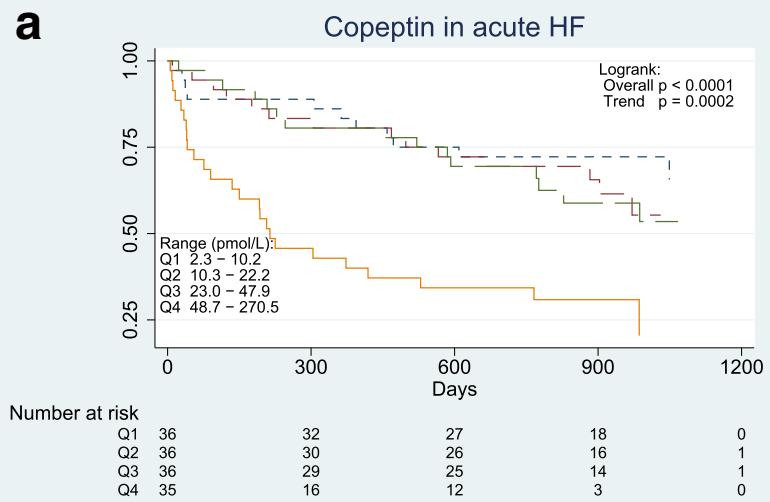

C

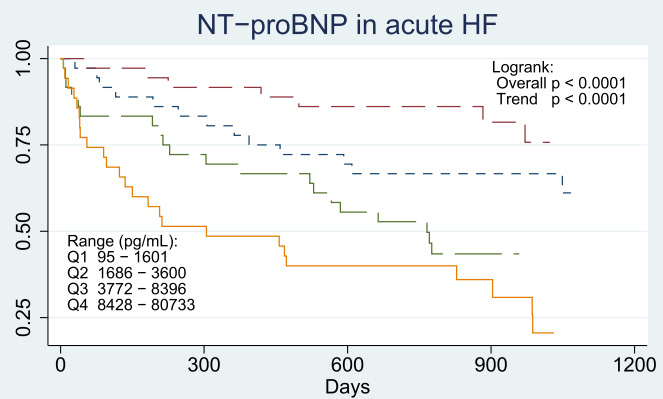

b

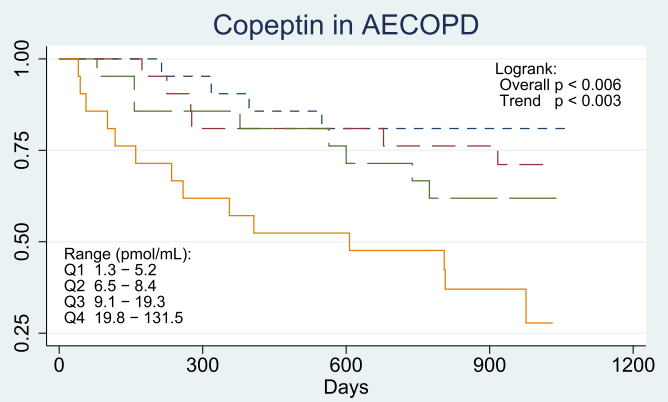

Number at risk

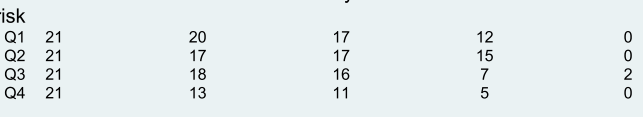

d

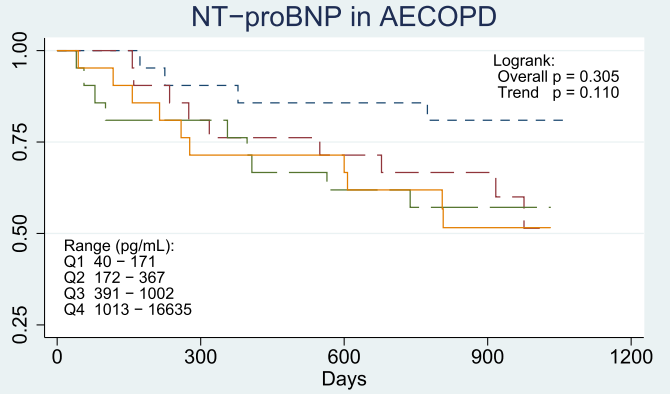

Number at risk

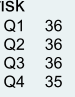

30
33
26
18
20
16
8
7

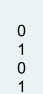

0
1
0
1

Number at risk

$\begin{array}{rlll}\text { risk } & & & \\ \text { Q1 } & 21 & 19 & 18 \\ \text { Q2 } & 21 & 17 & 15 \\ \text { Q3 } & 21 & 17 & 13 \\ \text { Q4 } & 21 & 15 & 15\end{array}$

18
15
13
15

14
10
5
10

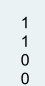

Quartiles (Q):

$-----$

$---2$

$-3$

4

Fig. 2 Kaplan-Meier survival plots stratified by biomarker quartiles for (a) copeptin in acute HF, (b) copeptin in AECOPD, (c) NT-proBNP in acute HF, and (d) NT-proBNP in AECOPD

COPD in the acute setting we categorized unselected patients with acute dyspnea by established guidelines under the scrutiny of two experts working independently and analyzed the prognostic properties of copeptin in AECOPD and acute HF separately. From the results of our study we confirm that copeptin is a strong predictor of two-year mortality in AECOPD independently of other pulmonary and cardiac risk factors.

The prognostic utility of natriuretic peptides in COPD is controversial. In agreement with the results from one previous study [31] we did not find any predictive value of NT-proBNP regarding mortality in AECOPD. Nevertheless, other studies have indicated that NT-proBNP could be a useful prognostic marker in COPD [32, 33]. The conflicting results concerning the prognostic value of NT-proBNP in different COPD cohorts may relate to misclassification of diagnosis or differing prevalence of cardiac complications and comorbidities associated with NT-proBNP and mortality, such as pulmonary hypertension [34], cor pulmonale [35], and left ventricular dysfunction [36-38]. As no specific index can be used to diagnose AECOPD or acute HF [26, 39]. we stratified our patients according to the diagnosis made by an adjudication committee, which is considered to be the "goldstandard" strategy in order to avoid misclassification. In addition, our adjudication committee classified patients more uniformly than previous adjudication committees in similar studies [40, 41]. In our AECOPD cohort, only nine patients $(11 \%)$ had a history of heart failure and the median LVEF was normal (60\% [Q1-3 50-60]), indicating low prevalence of cardiac dysfunction. Thus, our results show that copeptin is a strong prognostic marker independent of cardiac pathology and support the theory that the prognostic value of NT-proBNP in COPD is related to cardiac complications and comorbidities.

As the ACE 2 Study was moderate in size, the negative result for NT-proBNP in AECOPD could also be explained by low statistical power. However, no trend for increasing mortality by NT-proBNP categories was observed in Kaplan-Meier survival plots (Fig. 2). We could not find a statistical significant difference between the prognostic accuracy of copeptin and NT-proBNP by 
Table 2 Multivariate Cox proportional regression analysis for long-term mortality

\begin{tabular}{|c|c|c|c|c|}
\hline & \multicolumn{2}{|c|}{ Acute exacerbation of COPD $(n=84)$} & \multicolumn{2}{|l|}{$\begin{array}{l}\text { Acute HF } \\
(n=143) \\
\end{array}$} \\
\hline & $\mathrm{HR}(95 \% \mathrm{Cl})$ & $P$ & HR $(95 \% \mathrm{Cl})$ & $P$ \\
\hline \multicolumn{5}{|l|}{ Basic risk factors } \\
\hline Age (per year) & n.s. & & $1.04(1.01-1.07)$ & 0.009 \\
\hline BMI (per $\mathrm{kg} / \mathrm{m}^{2}$ ) & $0.90(0.84-0.96)$ & 0.002 & $0.94(0.89-1.01)$ & 0.032 \\
\hline Mean arterial pressure (per 5 mmHg) & n.s. & & $0.99(0.97-1.00)$ & 0.025 \\
\hline Diabetes mellitus (yes vs. no) & n.s. & & $2.56(1.50-4.36)$ & 0.001 \\
\hline COPD (yes vs. no) & n.s. & & $1.75(1.04-2.92)$ & 0.035 \\
\hline $\mathrm{K}^{+}($per $\mathrm{mmol} / \mathrm{l})$ & n.s. & & $1.88(1.21-2.92)$ & 0.005 \\
\hline In C-reactive protein (per log unit) & n.s. & & $1.21(1.01-1.46)$ & 0.042 \\
\hline \multicolumn{5}{|l|}{ Biomarkers adjusted for basic factors } \\
\hline In Copeptin (per log unit) & $1.72(1.21-2.45)$ & 0.003 & $1.61(1.25-2.09)$ & $<0.001$ \\
\hline In NT-proBNP (per log unit) & $1.12(0.88-1.42)$ & 0.373 & $1.62(1.27-2.06)$ & $<0.001$ \\
\hline In hs-TnT (per log unit) & $1.36(0.88-2.11)$ & 0.164 & $1.32(1.03-1.69)$ & 0.027 \\
\hline \multicolumn{5}{|c|}{ Biomarkers adjusted for basic factors and each other } \\
\hline In Copeptin (per log unit) & $1.79(1.20-2.66)$ & 0.004 & $1.30(0.96-1.76)$ & 0.091 \\
\hline In NT-proBNP (per log unit) & $0.94(0.71-1.24)$ & 0.668 & $1.43(1.07-1.89)$ & 0.014 \\
\hline
\end{tabular}

Median follow-up time period: 813 [Q1-3356-996] days

Abbreviations: BMI Body mass index, $C l$ confidence interval, COPD chronic obstructive pulmonary disease, $h s-T n T$ high sensitivity troponin T, HR hazard ratio, n.s not statistically significant, NT-proBNP N-terminal pro-B-type natriuretic peptide, vs. versus

ROC-AUC in AECOPD, in contrast to the results obtained by Cox proportional hazard regression analysis, but this inconsistency probably relates to inferior statistical properties when comparing predictors by ROCAUC as opposed to regression analysis [42]. NRI analyses found copeptin, but not NT-proBNP, to reclassify a significant proportion of patients into their correct risk strata on top of a basic clinical model. This emphasizes the superior prognostic value of copeptin over NTproBNP levels in AECOPD. Accordingly; our data support copeptin as the preferred prognostic biomarker concerning mortality in patients with AECOPD.

The prognostic accuracies of copeptin and NTproBNP seem comparable in HF. In previous studies of chronic HF, copeptin has been associated with shortand long-term prognostic outcomes independently of
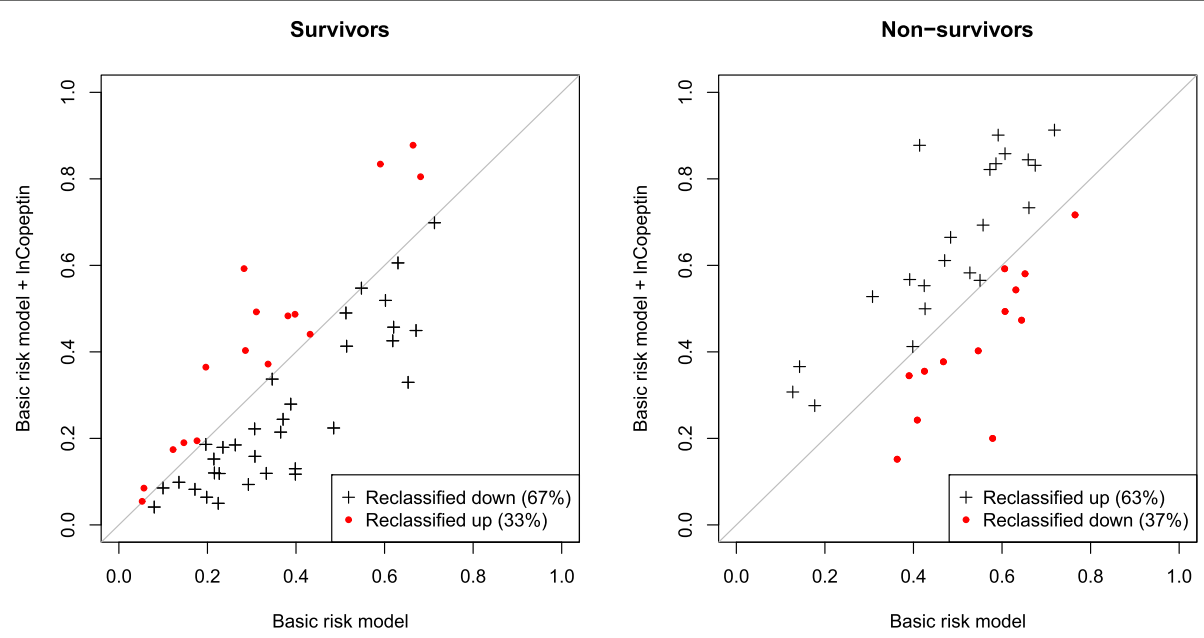

Fig. 3 Risk reclassification among AECOPD patients. Estimated risk of death for each patient by the basic model ( $x$-axis) and the enhanced model that also included copeptin (y-axis). "Reclassified up" or "reclassified down" represents an increased or decreased risk of death predicted by the enhanced model as compared to the basic model. The prediction model is improved when survivors are reclassified down, and non-survivors are reclassified up 
other risk factors including natriuretic peptides, and in some of these studies copeptin also predicted the outcome with higher accuracy than BNP and NTproBNP [16-18]. In our study, we found copeptin to be a strong predictor of mortality in acute HF patients independently of basic risk factors, but after adjustment for NT-proBNP the prognostic effect of copeptin by Cox regression was not significant (Table $2)$. In contrast, we did not find any significant difference between the prognostic accuracy of copeptin and NT-proBNP estimated by ROC-AUC or improvement in the overall predictive accuracy when NT-proBNP was added to the multivariate Cox regression model already including copeptin. The most likely explanation for these findings is that copeptin and NTproBNP carry much of the same prognostic information in acute HF. Hence, copeptin also appears to be a valid alternative to NT-proBNP with respect to mortality risk evaluation in acute HF.

NT-proBNP concentrations were superior to copeptin concentrations for the discrimination of acute HF from other causes of dyspnea in our patients. This is not surprising from a theoretical viewpoint as NT-proBNP is released mainly as a result of myocardial stretch [43], while copeptin release most likely is stimulated by the same mechanisms known to stimulate AVP secretion that are less specific for heart failure [6]. The finding that NT-proBNP is a better diagnostic marker of acute HF than copeptin is in line with our main finding that copeptin is a better prognostic marker than NT-proBNP in AECOPD patients.

The mechanisms responsible for increased copeptin release in AECOPD are unclear. The median concentration of copeptin found in AECOPD (8.8 [5.2-19.7]) is higher than what has been reported in healthy subjects recruited from the general population (3.7$4.2 \mathrm{pmol} / \mathrm{L})[12,44]$. A functioning osmotic regulation of copeptin release is indicated by the positive correlation between copeptin and $\mathrm{Na}^{+}$concentrations in our patients (Additional file 1: Figure S1), but contrary to what is expected under normal osmotic regulation [45], we found that copeptin is not clearly suppressed among AECOPD or acute HF patients with $\mathrm{Na}^{+}$concentrations $<137 \mathrm{mmol} / \mathrm{L}$ (7.6 [2.7-16.0] and 18.2 [6.3-52.6] pmol/L, respectively). The missing suppression of copeptin among hyponatremic patients suggest the presence of an non-osmotic stimulation of copeptin and AVP release that could also explain the high prevalence of hyponatremia in AECOPD (27\%) and acute HF (20\%) previously documented in the ACE 2 Study [23]. In HF patients, arterial underfilling is a strong stimulant of non-osmotic copeptin and AVP release via baroreceptors [7], but this pathway seem less likely in AECOPD patients with close to normal cardiac function. Another relevant question is whether the concentration of copeptin increases during AECOPD compared to stable-state COPD. Interestingly, one previous study of COPD patients could not find any difference between copeptin levels in stable-state and exacerbations [29]. We were, however, not able to explore this question further as stable-state copeptin measures were not included in the ACE 2 study design. Clearly, further studies are needed to explore the mechanisms that stimulate copeptin and AVP secretion in COPD.

\section{Conclusion}

Copeptin is a strong prognostic marker in patients with acute exacerbation of chronic obstructive pulmonary disease (AECOPD) and acute heart failure (HF), while NT-proBNP concentrations predict mortality only among patients with acute HF. Accordingly, copeptin could be preferable to NT-proBNP for risk stratification in AECOPD and mixed populations that include both AECOPD and acute HF patients. Copeptin concentrations are significantly higher in patients with acute HF compared to other etiologies of acute dyspnea, but NTproBNP is superior to copeptin for diagnosing HF in the acute setting.

\section{Additional file}

Additional file 1: Table S1. Linear regression for log (In) copeptin ( $n=314$ ). Table S2. Univariate proportional Cox regression analysis. Table S3. Multivariate proportional Cox regression analysis for the nonHF, non-AECOPD group. Figure S1. Correlation between copeptin and $\mathrm{Na}^{+}$. (DOCX $\left.267 \mathrm{~kb}\right)$

\section{Abbreviations}

ACE Study: Akershus Cardiac Examination Study; AECOPD: Acute exacerbation of chronic obstructive pulmonary disease; Cl: Confidence interval; CKD-EPI: Chronic Kidney Disease Epidemiology Collaboration; COPD: Chronic obstructive pulmonary disease; CRP: C-reactive protein; eGFR: estimated glomerular filtration rate; GOLD: Global initiative for Chronic Obstructive Lung Disease; HF: Heart failure; HR: Hazard ratio; hs-TnT: Highsensitivity troponin T; LVEF: Left ventricular ejection fraction; NRI: Net reclassification index; NT-proBNP: N-terminal pro-hormone of B-type natriuretic peptide; Q: Quartile; ROC-AUC: Area under receiver operating curves; SD: Standard deviation; Vs: versus

\section{Acknowledgments}

We would like to acknowledge the contribution by the Clinical Trail Unit, Division of Medicine, Akershus University Hospital and Vigdis Bakkelund, BSC; Marit Holmefjord Pedersen, BSc; and Annika Lorentzen, BSc with all aspects of the ACE 2 Study. We also thank physicians and nurses in the Division of Medicine and acknowledge the Department of Medical Biochemistry, Akershus University Hospital for laboratory analyses.

\section{Funding}

Akershus University Hospital and the Research Council of Norway funded the ACE 2 Study. Thermo Fisher Scientific, Clinical Diagnostics, BRAHMS GmbH 16,761 Hennigsdorf, Germany, Tel: +49-3302 - 883 - 0 provided copeptin reagents free of charge for this study. NT-proBNP kits were supplied at reduced price by Roche Diagnostics. The sponsors had no role in any of the following: design and conduct of the study, collection, 
management, analysis and interpretation of the data, or preparation, review and approval of the manuscript.

\section{Availability of data and materials}

Due to ethical restrictions related to patient privacy, raw data are only available upon request, subject to standard approval from the data custodian: Dr. Helge Røsjø (helge.rosjo@medisin.uio.no).

\section{Authors' contributions}

JAW and TO had full access to all data in the study and take responsibility for the integrity of the data and the accuracy of the data analysis. GC, TO, and $H R$ designed the clinical studies. JB, ADH, and $H R$ were engaged in data acquisition, HS was responsible for the biochemical analyses, while JAW, JB, IF, SN, HR, and TO contributed to analysis and/or interpretation of data. All authors have critically revised the manuscript and have approved the final version.

\section{Ethics approval and consent to participate}

The ACE 2 study was approved by the Norwegian Regional Committees for Medical and Health Research Ethics (REC) South East (\#5.2008.2832) and conducted in agreement with the Declaration of Helsinki. All participants provided written informed consent prior to study enrolment.

\section{Consent for publication}

Not applicable.

\section{Competing interests}

JAW has received consultancy and speaker honoraria from Otsuka Pharmaceuticals Europe Ltd. TO has received consultancy and speaker honoraria from Abbott Diagnostics and Roche Diagnostics. HR has received speaker honoraria from Novartis. All other authors declare no competing interests.

\section{Publisher's Note}

Springer Nature remains neutral with regard to jurisdictional claims in published maps and institutional affiliations.

\section{Author details}

${ }^{1}$ Division of Medicine, Akershus University Hospital, Lørenskog, Norway. ${ }^{2}$ Institute of Clinical Medicine, University of Oslo, Oslo, Norway. ${ }^{3}$ Institute for Experimental Medical Research, Oslo University Hospital and University of Oslo, Oslo, Norway. ${ }^{4}$ Division of Diagnostics and Technology, Akershus University Hospital, Lørenskog, Norway. ${ }^{5}$ Bioinformatics Core Facility, Institute for Medical Informatics, Oslo University Hospital and University of Oslo, Oslo, Norway.

Received: 29 May 2017 Accepted: 11 October 2017

Published online: 03 November 2017

\section{References}

1. de Lemos JA, Morrow DA, Bentley JH, Omland T, Sabatine MS, McCabe CH, Hall C, Cannon CP, Braunwald E. The prognostic value of B-type natriuretic peptide in patients with acute coronary syndromes. N Engl J Med. 2001;345: 1014-21.

2. Bettencourt P. NT-proBNP and BNP: biomarkers for heart failure management. Eur J Heart Fail. 2004;6:359-63.

3. Kragelund C, Gronning B, Kober L, Hildebrandt P, Steffensen R. N-terminal pro-B-type natriuretic peptide and long-term mortality in stable coronary heart disease. N Engl J Med. 2005:352:666-75.

4. Koutsokera A, Stolz D, Loukides S, Kostikas K. Systemic biomarkers in exacerbations of COPD: the evolving clinical challenge. Chest. 2012;141:396-405

5. Christ-Crain M, Fenske W. Copeptin in the diagnosis of vasopressindependent disorders of fluid homeostasis. Nat Rev Endocrinol. 2016;

6. Schrier RW, Berl T, Anderson RJ. Osmotic and nonosmotic control of vasopressin release. Am J Phys. 1979;236:F321-32.

7. Robertson GL, Shelton RL, Athar S. The osmoregulation of vasopressin. Kidney Int. 1976;10:25-37.

8. Farber MO, Roberts LR, Weinberger MH, Robertson GL, Fineberg NS, Manfredi F. Abnormalities of sodium and $\mathrm{H} 2 \mathrm{O}$ handling in chronic obstructive lung disease. Arch Intern Med. 1982;142:1326-30.
9. de Leeuw PW, Dees A. Fluid homeostasis in chronic obstructive lung disease. Eur Respir J Suppl. 2003;46:33s-40s.

10. Itoi K, Jiang YQ, Iwasaki Y, Watson SJ. Regulatory mechanisms of corticotropin-releasing hormone and vasopressin gene expression in the hypothalamus. J Neuroendocrinol. 2004;16:348-55.

11. Preibisz JJ, Sealey JE, Laragh JH, Cody RJ, Weksler BB. Plasma and platelet vasopressin in essential hypertension and congestive heart failure. Hypertension. 1983;5:1129-38.

12. Morgenthaler NG, Struck J, Alonso C, Bergmann A. Assay for the measurement of copeptin, a stable peptide derived from the precursor of vasopressin. Clin Chem. 2006:52:112-9.

13. Struck J, Morgenthaler NG, Bergmann A. Copeptin, a stable peptide derived from the vasopressin precursor, is elevated in serum of sepsis patients. Peptides. 2005;26:2500-4.

14. Morgenthaler NG, Muller B, Struck J, Bergmann A, Redl H, Christ-Crain M. Copeptin, a stable peptide of the arginine vasopressin precursor, is elevated in hemorrhagic and septic shock. Shock. 2007:28:219-26.

15. Khan SQ, Dhillon OS, O'Brien RJ, Struck J, Quinn PA, Morgenthaler NG, Squire IB, Davies JE, Bergmann A, Ng LL. C-terminal provasopressin (copeptin) as a novel and prognostic marker in acute myocardial infarction: Leicester acute myocardial infarction peptide (LAMP) study. Circulation. 2007;115:2103-10

16. Stoiser B, Mortl D, Hulsmann M, Berger R, Struck J, Morgenthaler NG, Bergmann A, Pacher R. Copeptin, a fragment of the vasopressin precursor, as a novel predictor of outcome in heart failure. Eur J Clin Investig. 2006;36:771-8.

17. Neuhold S, Huelsmann M, Strunk G, Stoiser B, Struck J, Morgenthaler NG, Bergmann A, Moertl D, Berger R, Pacher R. Comparison of copeptin, B-type natriuretic peptide, and amino-terminal pro-B-type natriuretic peptide in patients with chronic heart failure: prediction of death at different stages of the disease. J Am Coll Cardiol. 2008;52:266-72.

18. Voors AA, von Haehling S, Anker SD, Hillege HL, Struck J, Hartmann O, Bergmann A, Squire I, van Veldhuisen DJ, Dickstein K, Investigators O. Cterminal provasopressin (copeptin) is a strong prognostic marker in patients with heart failure after an acute myocardial infarction: results from the OPTIMAAL study. Eur Heart J. 2009;30:1187-94.

19. Muller B, Morgenthaler N, Stolz D, Schuetz P, Muller C, Bingisser R, Bergmann A, Tamm M, Christ-Crain M. Circulating levels of copeptin, a novel biomarker, in lower respiratory tract infections. Eur J Clin Investig. 2007:37:145-52.

20. Potocki M, Breidthardt T, Mueller A, Reichlin T, Socrates T, Arenja N, Reiter M, Morgenthaler NG, Bergmann A, Noveanu M, et al. Copeptin and risk stratification in patients with acute dyspnea. Crit Care. 2010;14:R213.

21. Ottesen AH, Louch WE, Carlson CR, Landsverk OJ, Kurola J, Johansen RF, Moe MK, Aronsen JM, Hoiseth AD, Jarstadmarken $\mathrm{H}$, et al. Secretoneurin is a novel prognostic cardiovascular biomarker associated with cardiomyocyte calcium handling. J Am Coll Cardiol. 2015;65:339-51.

22. Rosjo H, Dahl MB, Jorgensen M, Roysland R, Brynildsen J, Cataliotti A, Christensen G, Hoiseth AD, Hagve TA, Omland T. Influence of glycosylation on diagnostic and prognostic accuracy of $\mathrm{N}$-terminal pro-B-type natriuretic peptide in acute dyspnea: data from the Akershus cardiac examination 2 study. Clin Chem. 2015;61:1087-97.

23. Winther JA, Brynildsen J, Hoiseth AD, Folling I, Brekke PH, Christensen G, Hagve TA, Verbalis JG, Omland T, Rosjo H. Prevalence and prognostic significance of Hyponatremia in patients with acute exacerbation of chronic obstructive pulmonary disease: data from the Akershus cardiac examination (ACE) 2 study. PLoS One. 2016;11:e0161232.

24. Roysland R, Pervez MO, Pedersen MH, Brynildsen J, Hoiseth AD, Hagve TA, Rosjo H, Omland T. Diagnostic and prognostic properties of Osteoprotegerin in patients with acute Dyspnoea: observations from the Akershus cardiac examination (ACE) 2 study. PLoS One. 2016;11:e0160182.

25. McMurray JJ, Adamopoulos S, Anker SD, Auricchio A, Bohm M, Dickstein K, Falk V, Filippatos G, Fonseca C, Gomez-Sanchez MA, et al. ESC guidelines for the diagnosis and treatment of acute and chronic heart failure 2012: the task force for the diagnosis and treatment of acute and chronic heart failure 2012 of the European Society of Cardiology. Developed in collaboration with the heart failure association (HFA) of the ESC. Eur Heart J. 2012;33:1787-847.

26. Global Initiative for Chronic Obstructive Lung Disease (GOLD). Global strategy for Diagnosis, Management and Prevention of Chronic Pulmonary Disease [http://www.goldcopd.org/]. 
27. Stolz D, Christ-Crain M, Morgenthaler NG, Leuppi J, Miedinger D, Bingisser R, Muller C, Struck J, Muller B, Tamm M. Copeptin, C-reactive protein, and procalcitonin as prognostic biomarkers in acute exacerbation of COPD. Chest. 2007;131:1058-67.

28. Freemantle N, Calvert M, Wood J, Eastaugh J, Griffin C. Composite outcomes in randomized trials: greater precision but with greater uncertainty? JAMA. 2003;289:2554-9.

29. Boeck L, Soriano JB, Brusse-Keizer M, Blasi F, Kostikas K, Boersma W, Milenkovic B, Louis R, Lacoma A, Djamin R, et al. Prognostic assessment in COPD without lung function: the B-AE-D indices. Eur Respir J. 2016;47:1635-44.

30. Dres M, Hausfater P, Foissac F, Bernard M, Joly LM, Sebbane M, Philippon AL, Gil-Jardine C, Schmidt J, Maignan M, et al. Mid-regional proadrenomedullin and copeptin to predict short-term prognosis of COPD exacerbations: a multicenter prospective blinded study. Int J Chron Obstruct Pulmon Dis. 2017:12:1047-56.

31. Stolz D, Breidthardt T, Christ-Crain M, Bingisser R, Miedinger D, Leuppi J, Mueller B, Tamm M, Mueller C. Use of B-type natriuretic peptide in the risk stratification of acute exacerbations of COPD. Chest. 2008;133:1088-94.

32. Chang CL, Robinson SC, Mills GD, Sullivan GD, Karalus NC, McLachlan JD, Hancox RJ. Biochemical markers of cardiac dysfunction predict mortality in acute exacerbations of COPD. Thorax. 2011;66:764-8.

33. Hoiseth AD, Omland T, Hagve TA, Brekke PH, Soyseth V. NT-proBNP independently predicts long term mortality after acute exacerbation of COPD - a prospective cohort study. Respir Res. 2012;13:97.

34. Leuchte HH, Baumgartner RA, Nounou ME, Vogeser M, Neurohr C, Trautnitz $\mathrm{M}$, Behr J. Brain natriuretic peptide is a prognostic parameter in chronic lung disease. Am J Respir Crit Care Med. 2006;173:744-50.

35. Bozkanat E, Tozkoparan E, Baysan O, Deniz O, Ciftci F, Yokusoglu M. The significance of elevated brain natriuretic peptide levels in chronic obstructive pulmonary disease. J Int Med Res. 2005;33:537-44

36. Gariani K, Delabays A, Perneger TV, Agoritsas T. Use of brain natriuretic peptide to detect previously unknown left ventricular dysfunction in patients with acute exacerbation of chronic obstructive pulmonary disease. Swiss Med Wkly. 2011;141:w13298.

37. Inoue $Y$, Kawayama $\mathrm{T}$, Iwanaga $\mathrm{T}$, Aizawa H. High plasma brain natriuretic peptide levels in stable COPD without pulmonary hypertension or cor pulmonale. Intern Med. 2009:48:503-12.

38. Abroug F, Ouanes-Besbes L, Nciri N, Sellami N, Addad F, Hamda KB, Amor AB, Najjar MF, Knani J. Association of left-heart dysfunction with severe exacerbation of chronic obstructive pulmonary disease: diagnostic performance of cardiac biomarkers. Am J Respir Crit Care Med. 2006;174:990-6.

39. Wang CS, FitzGerald JM, Schulzer M, Mak E, Ayas NT. Does this dyspneic patient in the emergency department have congestive heart failure? JAMA. 2005;294:1944-56.

40. Januzzi JL Jr, Camargo CA, Anwaruddin S, Baggish AL, Chen AA, Krauser DG, Tung R, Cameron R, Nagurney JT, Chae CU, et al. The N-terminal pro-BNP investigation of dyspnea in the emergency department (PRIDE) study. Am J Cardiol. 2005:95:948-54.

41. McCullough PA, Nowak RM, McCord J, Hollander JE, Herrmann HC, Steg PG, Duc P, Westheim A, Omland T, Knudsen CW, et al. B-type natriuretic peptide and clinical judgment in emergency diagnosis of heart failure: analysis from breathing not properly (BNP) multinational study. Circulation. 2002;106:416-22.

42. Vickers AJ, Cronin AM, Begg CB. One statistical test is sufficient for assessing new predictive markers. BMC Med Res Methodol. 2011;11:13.

43. Omland T, Hagve TA. Natriuretic peptides: physiologic and analytic considerations. Heart Fail Clin. 2009;5:471-87.

44. Reichlin T, Hochholzer W, Stelzig C, Laule K, Freidank H, Morgenthaler NG, Bergmann A, Potocki M, Noveanu M, Breidthardt T, et al. Incremental value of copeptin for rapid rule out of acute myocardial infarction. J Am Coll Cardiol. 2009;54:60-8.

45. Fenske W, Stork S, Blechschmidt A, Maier SG, Morgenthaler NG, Allolio B. Copeptin in the differential diagnosis of hyponatremia. J Clin Endocrinol Metab. 2009:94:123-9. 